\title{
Epidemiology and Survival after Spinal Meningioma Surgery: A Nationwide Population- Based Study
}

\author{
Vincent Jecko ${ }^{1}$, Joconde Weller ${ }^{2}$, Deborah Houston ${ }^{3}$, Charles Champeaux-Depond ${ }^{3,4,5}$ \\ ${ }^{I}$ Department of Neurosurgery, Pellegrin Hospital, Bordeaux, France \\ ${ }^{2}$ Agence Régionale de Santé, Saint Denis, France \\ ${ }^{3}$ Department of Neurosurgery, Institute of Neurological Sciences, Queen Elizabeth University Hospital, Glasgow, UK \\ ${ }^{4}$ Department of Neurosurgery, Lariboisière Hospital, Paris, France
}

${ }^{5}$ INSERM U1153, Statistic and Epidemiologic Research Center Sorbonne Paris Cité (CRESS), ECSTRRA Team, Université de Paris, Paris, France

Study Design: This is a cross-sectional nationwide descriptive observational and analytic retrospective study.

Purpose: This study aims to describe and assess survival after spinal meningioma (SM) surgery.

Overview of Literature: A few studies report a reduced survival after SM surgery.

Methods: The current study processed the Système National des Données de Santé (SNDS), the French national administrative medical database, to retrieve appropriate cases.

Results: This study identified 2,844 patients (79.1\% females) between 2008 and 2017 . The median age at surgery was 66 years (interquartile range [IOR], 56-75 years). Moreover, $95.9 \%$ of SMs were removed through posterior or posterolateral approaches, and $6.9 \%$ were epidural and $0.7 \%$ needed an associated spine stabilization. Benign meningioma represented $92.9 \%$, with $5 \%$ and $2.1 \%$ atypical and malignant, respectively. The median follow-up was 3.3 years (IOR, 3.1-3.5 years). Of the patients, $0.25 \%$ and $1.2 \%$ expired within a month and a year of surgery, respectively. At data collection, 225 patients $(7.9 \%)$ expired. The 5 -year overall survival (OS) probability was $90.1 \%(95 \% \mathrm{Cl}, 88.6 \%-91.7 \%)$. However, absolute excess risk of mortality after SM surgery was null, and the related standardized mortality ratio was $1(95 \% \mathrm{Cl}, 0.9-1.2 ; p=0.565)$. In the adjusted regression, age at surgery (hazard ratio [HR], 1.06 ; $95 \% \mathrm{Cl}, 1.04-1.07 ; p<0.001$ ), level of comorbidities (HR, 1.44; 95\% Cl, 1.34-1.54; $p<0.001$ ), neurofibromatosis type 2 (NF2; HR, 3.65; $95 \% \mathrm{Cl}, 1.28-10.39 ; p=0.0152)$, epidural SM (HR, 1.73; 95\% Cl, 1.09-2.75; $p=0.0206)$, and malignant meningioma (HR, 2.64; $95 \% \mathrm{Cl}$, $1.51-4.61 ; p<0.001)$ remained significantly associated to a reduced OS.

Conclusions: The SNDS is of great value in assessing SM incidence, associated mortality, and its predictors. OS after meningioma surgery is favorable but may be impaired for NF2 or older patients with a high level of comorbidities, epidural tumor, and malignant histopathology. SM surgery is not associated with an increased absolute excess mortality risk despite being performed on even more senior patients compared with intracranial meningioma.

Keywords: Spinal meningioma; Epidemiology; Survival; Relative survival; Predictors

Received Jun 1, 2021; Revised Jun 30, 2021; Accepted Jul 27, 2021

Corresponding author: Charles Champeaux-Depond

Department of Neurosurgery, Larbiboisière Hospital, 2, rue Ambroise-Paré, 75475 Paris Cedex 10, France

Tel: +33-1-49-95-6565, Fax: +33-1-4995-8155, E-mail: Charles.Champeaux@gmail.com 


\section{Introduction}

Meningiomas, thought to arise from the meningothelial cells of the arachnoid, are the most common primary intracranial extracerebral tumors, accounting for 36.8\%$37.6 \%$ according to the Central Brain Tumor Registry of the United States [1]. Those developed in the spine are less frequent compared with those encountered intracranially, representing about $5 \%-10 \%$ of all meningiomas $[2,3]$. Nonetheless, spinal meningioma (SM) is the most common intradural spine tumor with $30.7 \%$ with the main differential diagnosis of schwannoma [3]. SMs are usually sporadic, but few genetic diseases (e.g., neurofibromatosis type 2 [NF2]) are identified risk factors [4].

The 2016 World Health Organization (WHO) classification of tumors affecting the central nervous system recognizes three grades of meningiomas. The WHO grade I or benign meningiomas are the most common and have usually a good outcome $[2,5,6]$. The WHO grade III or malignant meningiomas are rare and aggressive neoplasms with a poor prognosis $[7,8]$. The behavior and outcome of atypical WHO grade II are intermediate $[9,10]$.

Management options include regular monitoring especially for incidental tumors, symptom control, surgical excision, external beam radiation therapy (EBRT), and occasional chemotherapy. However, tailored maximal resection is the treatment of choice for symptomatic SM. Further optimal management is difficult to establish, and the role of postoperative EBRT as standard adjuvant treatment remains controversial apart from the rare malignant forms [8-10].

SMs are slow-growing tumors and may cause various symptoms once they have reached a significant volume causing cord and roots compression depending on their localization (i.e., pain, sensory and sphincter disturbance, and motor weakness up to paraplegia). SMs are generally benign. Moreover, complete excision should be the goal of surgery, thus providing usually a cure to the patient and its symptoms improvement.

Administrative medical databases (AMDB) are massive repositories of collected healthcare data for various purposes. AMDB provide a variety of already stored data with a constant and often increasing ongoing collection process. They encompass a very large population and frequently the whole nation, ensuring high statistical power without biases related to the sample representativity. They can be used to conduct epidemiological studies and evalu- ate medical practices [11]. Around 3,000 patients are operated on for a meningioma each year in France, but only a fraction for SM.

This study aims to describe the epidemiology, assess the survival after SM surgery, and search for associated prognostic factors.

\section{Materials and Methods}

\section{Clinical material}

A cross-sectional nationwide descriptive observational and analytic retrospective study was performed using Système National des Données de Santé (SNDS). Incidental SM that was never operated on is not considered in this study, and only surgically treated SMs were considered. Data were extracted from the SNDS, the national French medico-administrative database. Patients who underwent the surgical resection of a meningioma between January 1, 2008, and December 31, 2017, were included. Cases were selected using an algorithm combining two variables as previously described: the type of the surgical procedure identified by the Common Classification of Medical Acts (CCAM) and the primary diagnosis following the 10th revision of the International Classification of Diseases (ICD-10) $[2,4,12]$. Benign meningiomas were considered as corresponding to the D32 ICD-10 codes, atypical to D42, and malignant to C70. The first recorded date of SM surgery was defined as the index date. Patients $<18$ years were excluded $(n=22)$. Progression was defined as any new treatment for meningioma recurrence, e.g., redo surgery, radiotherapy, or stereotactic radiosurgery. The Mortality-Related Morbidity Index (MRMI), predictive of all-cause mortality, and the Expenditure-Related Morbidity Index (ERMI), predictive of healthcare expenditure, were used to assess the global health-state severity [13].

\section{Statistical methods}

For cohort description, continuous variables are reported as medians and interquartile ranges (IQRs), and categorical variables are reported as frequencies and proportions. Survival was measured from the first date at meningioma surgery to the date of expiry or censored at the last followup. Follow-up time was calculated using the reverse Kaplan-Meier estimator method. In essence, no patient in the SNDS was lost to follow-up because those who 
expired were automatically registered as such in the database. To account for the lack of cause-specific survival, survival analysis of the SM patients cohort relative to the expected age- and gender-matched French general population was performed. The life table methodology was used to obtain estimates of relative survival (RS). All tests were two-sided, and statistical significance was defined with an alpha level of $0.05(p<0.05)$. Analysis was performed using the SAS Enterprise Guide ver. 7.15 HF8 (SAS Institute Inc., Cary, NC, USA) and the R programming language and software environment for statistical computing and graphics (ver. 4.1.0, 2021-5-18; The R Foundation for Statistical Computing, Vienna, Austria) [14]. Analyses were implemented using the packages relsurv and survexp. fr among others. Multiplicative regression models were achieved using the Andersen's multiplicative regression model, which is an extension of the Cox regression model using RS. The statistical program and workflow were written in R Markdown ver. 2 with RStudio (RStudio, Boston, MA, USA) for dynamic and reproducible research.

\section{Compliance with ethical standards}

This study was conducted following the ethical guidelines for epidemiological research under the ethical standards of the Helsinki Declaration (2008); the French data protection authority (CNIL; Commission Nationale de l'Informatique et des Libertés), an independent national ethical committee (authorization no., 2008538); and the RECORD (Reporting of studies Conducted using Observational Routinely-collected Data) guidelines for studies conducted using routinely collected health data and according to the SAMPL Guidelines $[15,16]$. Informed consent was not required because of the retrospective nature of the study. The SNDS encrypts patient personal information to protect patients' privacy and provides researchers with anonymous identification numbers.

\section{Results}

\section{Population description}

Within the SNDS, 2,844 patients who had SM surgery were identified between 2008 and 2017. Of the patients, $79.1 \%$ were females, and the median age at surgery was 66 years (IQR, 56-75 years). According to the MRMI, males had significantly more comorbidities compared with fe- males $(p<0.001)$. The level of comorbidity also increased with age $(p<0.001)$. In addition, $95.9 \%$ of the SM were removed through a posterior or posterior-lateral approach, and $6.9 \%$ were epidural and $0.7 \%$ need an associated spine stabilization.

Benign meningioma represented $92.9 \%$ with $5 \%$ and $2 \%$ atypical and malignant, respectively (Table 1 ). The median hospital stay was 8 days (IQR, 7-13 years). Of the patients, $59.1 \%$ and $24.8 \%$ were discharged at home and a in rehabilitation unit, respectively. In addition, others were discharged to another hospital department. Unsurprisingly, the patients' hospital stay was longer for those needing rehabilitation ( 7 days versus 12 days, $p<0.001)$ and for those with a higher level of comorbidities ( 8 days versus 11 days, $p<0.001)$. The patients discharged home were significantly younger compared with those transferred to a rehabilitation unit (62 years versus 72 years, $p<0.001$ ) and had significantly fewer comorbidities $(p<0.001)$. The median follow-up was 3.3 years (IQR, 3.1-3.5 years).

\section{Survival}

At data collection, 225 patients (7.9\%) expired. The median age at expiry was 80.2 years (IQR, $70.8-85.8$ years). Seven $(0.25 \%), 15(0.53 \%)$, and $35(1.23 \%)$ patients expired within the first year, the third year, and a year, respectively. The 5 -year OS was $90.1 \%$ (95\% confidence interval [CI], 88.6\%-91.7\%) (Fig. 1A).

For benign, atypical, and malignant meningiomas, the 5-year OS rates were $91.3 \%$ (95\% CI, 89.7\%-92.8\%), 86.6\% (95\% CI, 79.1\%-94.7\%), and 54.4\% (95\% CI, 40\%$74.1 \%)$, respectively $(p<0.001)$ (Fig. 1C).

In univariable Cox modeling, age at surgery (hazard ratio [HR], 1.07; 95\% CI, 1.05-1.08; $p<0.001$ ), MRMI (HR, 1.56; 95\% CI, 1.46-1.66; $p<0.001$ ), ERMI (HR, 1.11; 95\% CI, 1.09-1.13; $p<0.001)$, anterior or anterior-lateral approaches (HR, 1.63; 95\% CI, 0.99-2.67; $p=0.0526$ ), epidural SM (HR, 1.9; 95\% CI, 1.27-2.83; $p=0.00175)$, malignant meningioma (HR, 5.44; 95\% CI, 3.43-8.62; $p<0.001$ ), the need for EBRT (HR, 2.38; 95\% CI, 1.22-4.63; $p=0.011$ ), a prolonged hospital stay (HR, 1.02; 95\% CI, 1.01-1.02; $p<0.001$ ), and the need of a rehabilitation center (HR, $1.69 ; 95 \%$ CI, $1.29-2.21 ; p<0.001)$ were associated with a shorter OS (Table 2).

In the adjusted regression, age at surgery (HR, 1.06; 95\% CI, 1.04-1.07; $p<0.001$ ), level of comorbidities (HR, 1.44; 95\% CI, 1.34-1.54; $p<0.001$ ), NF2 (HR, 3.65; 95\% 


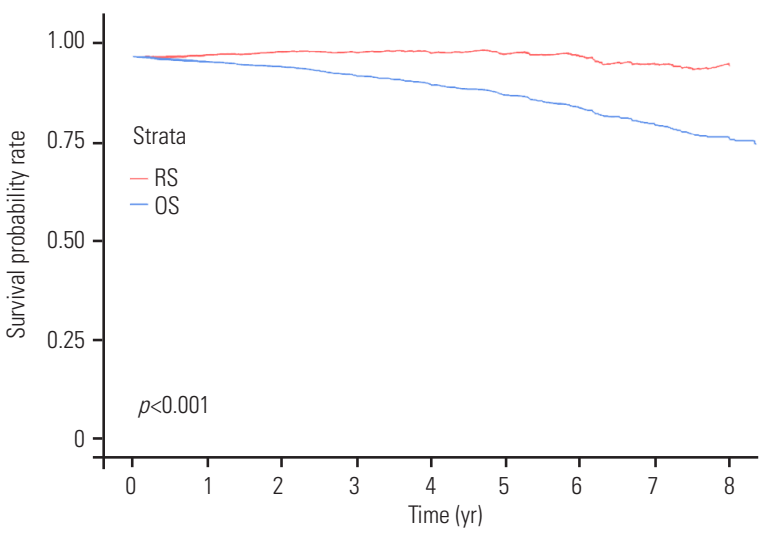

No. at risk
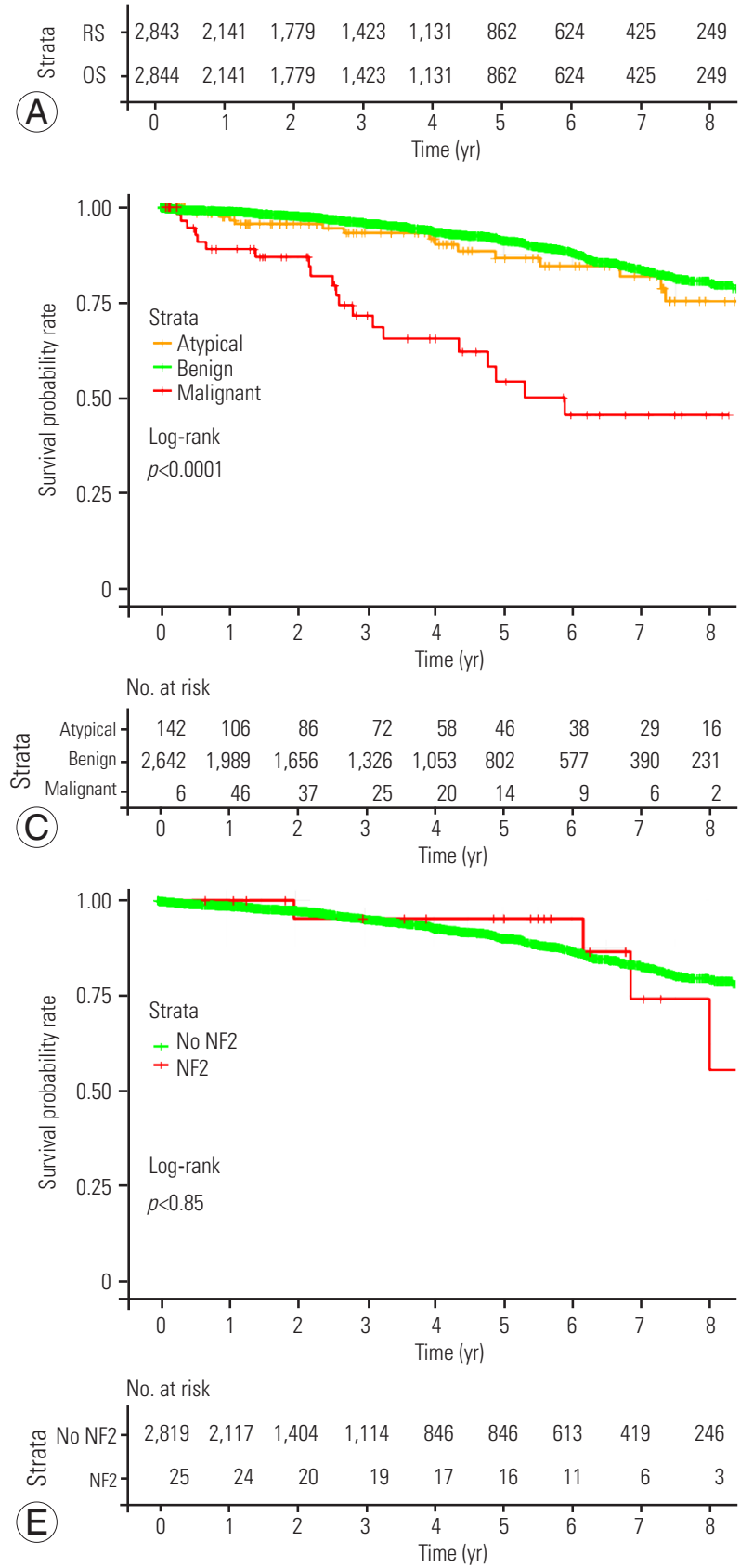
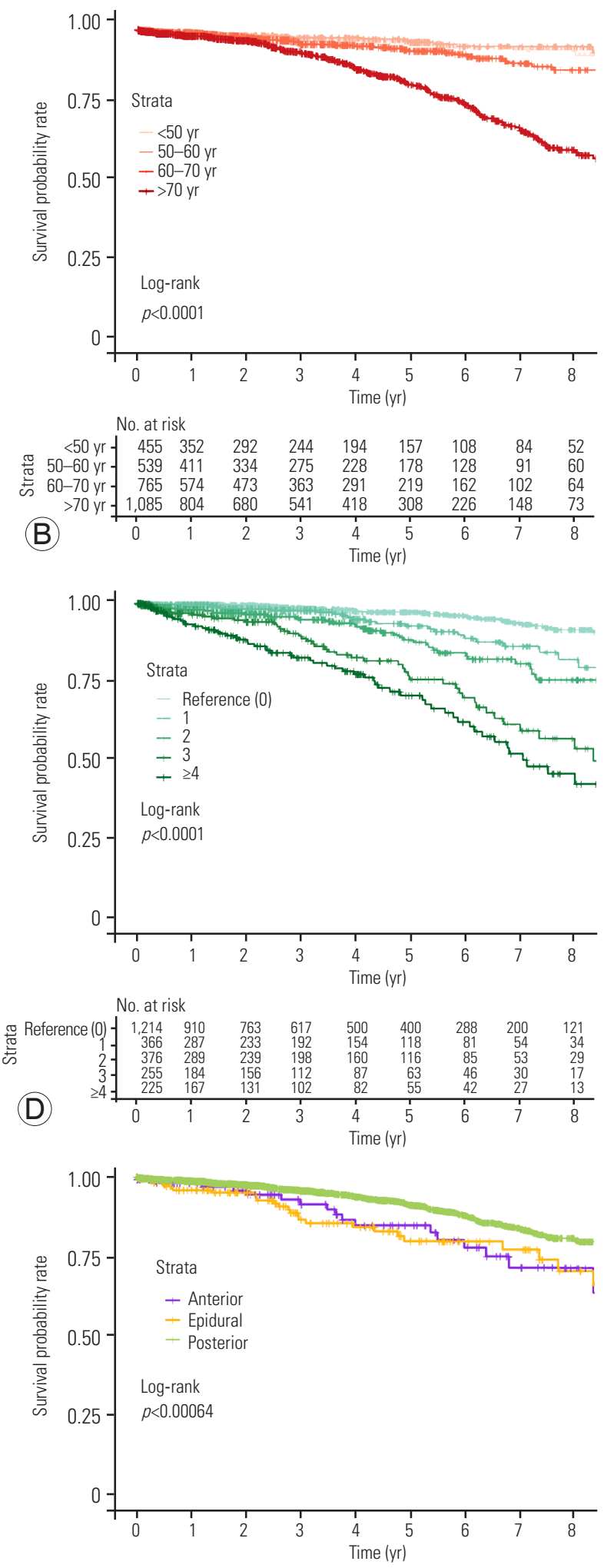

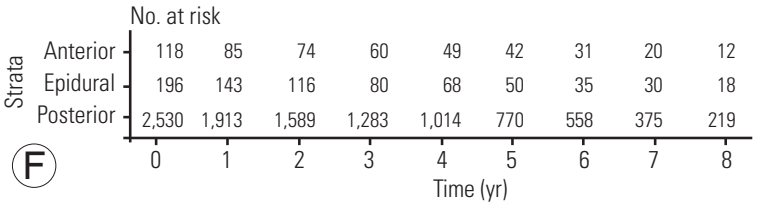

Fig. 1. Plots of Kaplan-Meier overall survival (OS) curves comparison. (A) OS and relative survival (RS). (B) OS by categories of age. (C) OS by grade. (D) OS by Mortality-Related Morbidity Index. (E) OS by neurofibromatosis type 2 (NF2). (F) OS by surgical technique and location. 
Table 1. Characteristics of the 28,778 patients

\begin{tabular}{|c|c|}
\hline Characteristic & Value \\
\hline Gender (female) & $2,251(79.1)$ \\
\hline Median age at surgery (yr) & $66(56-75)$ \\
\hline \multicolumn{2}{|l|}{ Age at surgery (yr) } \\
\hline$<50$ & $455(16.0)$ \\
\hline$>50-59$ & $539(19.0)$ \\
\hline$>60-<69$ & $765(26.9)$ \\
\hline$>70$ & $1,085(38.2)$ \\
\hline Neurofibromatosis type 2 & $25(0.9)$ \\
\hline Mortality-Related Morbidity Index & $1(0-2)$ \\
\hline Expenditure-Related Morbidity Index ${ }^{a)}$ & $2(0-9)$ \\
\hline \multicolumn{2}{|l|}{ Surgical technique } \\
\hline Posterior or posterior lateral approach & $2,530(89.0)$ \\
\hline Anterior or anterior lateral approach & $108(3.8)$ \\
\hline Epidural meningioma, posterior approach & $186(6.5)$ \\
\hline Epidural meningioma posterior approach with spinal fusion & $10(0.4)$ \\
\hline Anterior or anterior lateral approach with spinal fusion & $10(0.4)$ \\
\hline Posterior approach & $2,726(95.9)$ \\
\hline Anterior approach & $118(4.1)$ \\
\hline Epidural meningioma & $196(6.9)$ \\
\hline Spinal fixation & $20(0.7)$ \\
\hline \multicolumn{2}{|l|}{ Tumor grading } \\
\hline Benign & $2,642(92.9)$ \\
\hline Atypical & $142(5.0)$ \\
\hline Malignant & $60(2.1)$ \\
\hline Aggressive meningioma & $202(7.1)$ \\
\hline Redo surgery for recurrence & $100(3.5)$ \\
\hline Radiotherapy or stereotactic radiosurgery & $30(1.1)$ \\
\hline Hospital stay (day) & $8(7-13)$ \\
\hline Transfer to rehabilitation after spinal meningioma surgery & $705(24.8)$ \\
\hline \multicolumn{2}{|l|}{ Death } \\
\hline Within the postoperative month & $7(0.2)$ \\
\hline Within the 3 postoperative months & $15(0.5)$ \\
\hline At last follow-up & $225(7.9)$ \\
\hline
\end{tabular}

Values are presented as number (\%) or median (interquartile range).

${ }^{a}$ Indices computed using exclusively condition-related weights.
Table 2. Univariable Cox regression for spinal meningioma overall survival

\begin{tabular}{lcc}
\multirow{2}{*}{ Variable } & \multicolumn{2}{c}{ Overall survival } \\
\cline { 2 - 3 } & HR $(95 \% \mathrm{CI})$ & $p$-value \\
Gender (female) & $0.79(0.59-1.06)$ & 0.12 \\
Age at surgery & $1.07(1.05-1.08)$ & $<0.001$
\end{tabular}

Age at surgery (yr)

\begin{tabular}{|ccc|}
\hline$<50$ & Reference & \\
\hline$>50-60$ & $0.93(0.46-1.87)$ & 0.83 \\
\hline$>60-<70$ & $1.87(1.03-3.39)$ & 0.04 \\
\hline$>70$ & $5.57(3.28-9.45)$ & $<0.001$ \\
\hline Neurofibromatosis type 2 & $1.1(0.41-2.97)$ & 0.85 \\
\hline Mortality-Related Morbidity Index & $1.56(1.46-1.66)$ & $<0.001$ \\
\hline $\begin{array}{l}\text { Expenditure-Related Morbidity Index (continuous) } \\
\text { Surgical technique }\end{array}$ & $1.11(1.09-1.13)$ & $<0.001$ \\
\hline $\begin{array}{l}\text { Posterior or posterior lateral approach } \\
\text { Anterior or anterior lateral approach }\end{array}$ & \\
\hline $\begin{array}{l}\text { Epidural meningioma, posterior approach } \\
\text { Epidural meningioma posterior approach with }\end{array}$ & $1.83(1.1-3.05)$ & 0.02 \\
\hline $\begin{array}{l}\text { spinal fusion } \\
\text { Anterior or anterior lateral approach with spinal }\end{array}$ & $0.92(0.82-13.3)$ & 0.09 \\
\hline fusion & & \\
\hline
\end{tabular}

Surgical technique

\begin{tabular}{|lcc|}
\hline \multicolumn{1}{|c|}{ Posterior or posterior lateral approach } & Reference & \\
\hline \multicolumn{1}{|c|}{ Anterior or anterior lateral approach fusion } & $1.73(1.05-2.84)$ & 0.03 \\
\hline \multicolumn{1}{|c|}{ Epidural SM fusion } & $1.96(1.31-2.94)$ & $<0.001$ \\
\hline Posterior approach & $0.61(0.37-1.01)$ & 0.05 \\
\hline Anterior approach & $1.63(0.99-2.67)$ & 0.05 \\
\hline Epidural meningioma & $1.9(1.27-2.83)$ & $<0.001$ \\
\hline Spinal fixation & $1.63(0.52-5.1)$ & 0.4 \\
\hline Tumor grading & & \\
\hline \multicolumn{1}{|c|}{ Benign } & Reference & \\
\hline \multicolumn{1}{|c|}{ Atypical } & $1.33(0.8-2.22)$ & 0.27 \\
\hline Malignant & $5.44(3.43-8.62)$ & $<0.001$ \\
\hline Redo surgery for recurrence & $0.79(0.43-1.45)$ & 0.45 \\
\hline Radiotherapy or stereotactic radiosurgery & $2.38(1.22-4.63)$ & 0.01 \\
\hline Length of hospital stay & $1.02(1.01-1.02)$ & $<0.001$ \\
\hline Transfer to rehabilitation after SM surgery & $1.69(1.29-2.21)$ & $<0.001$ \\
\hline
\end{tabular}

$p$-values displayed in bold reached the statistical significance. $\mathrm{HR}$, hazard ratio; $\mathrm{Cl}$, confidence interval; SM, spinal meningioma.

was $100.6 \%$ (95\% CI, 98.6-102.5), suggesting that meningioma did not contribute to the overall mortality (Fig. 1F). Absolute excess risk of mortality after SM surgery was null, and the related standardized mortality ratio was 1 (95\% CI, $0.9-1.2 ; p=0.565)$. 


\begin{tabular}{|c|c|c|}
\hline \multirow{2}{*}{ Variable } & \multicolumn{2}{|c|}{ Overall survival } \\
\hline & $\mathrm{HR}(95 \% \mathrm{Cl})$ & $p$-value \\
\hline Age at surgery & $1.06(1.04-1.07)$ & $<0.001$ \\
\hline Neurofibromatosis type 2 & $3.65(1.28-10.39)$ & 0.02 \\
\hline Mortality-Related Morbidity Index & $1.44(1.34-1.54)$ & $<0.001$ \\
\hline \multicolumn{3}{|l|}{ Surgical technique } \\
\hline Posterior or posterior lateral approach & Reference & \\
\hline Epidural spinal meningioma fusion & $1.73(1.09-2.75)$ & 0.02 \\
\hline \multicolumn{3}{|l|}{ Tumor grading } \\
\hline Grade I (benign) & Reference & \\
\hline Grade III (malignant) & $2.64(1.51-4.61)$ & $<0.001$ \\
\hline
\end{tabular}

$p$-values displayed in bold reached the statistical significance. $\mathrm{HR}$, hazard ratio; $\mathrm{Cl}$, confidence interval.

\section{Discussion}

This work presents a unique modern population-based analysis of SM patients, which represent $9.8 \%$ of all the meningiomas of the current database versus $7.7 \%, 7.9 \%$, and solely $4.25 \%$ of Brodbelt et al. [17], Westwick and Shamji [3], and Cao et al. [18], respectively. Compared to their intracranial counterparts, SM occurred even more frequently in women $(79.1 \%$ versus $74.6 \%, p<0.001)$ at a much older age (66 years [IQR, 56-75] versus 58 years [IQR, 48-67], $p<0.001)$. However, no satisfactory reason has been provided to explain these contrasts even if differences in hormone responsiveness and genomic makeup have been suggested $[3,17]$. The current findings are similar to those of Cao et al. [18], who analyzed the Surveillance, Epidemiology, and End Results (SEER) database. An age-adjusted incidence rate of 0.37 cases per 1,000,000 person-years, the greatest incidence in the 60- to 69-year group, and also a female preponderance with a gender ratio of 4 versus 3.8 in the current study were found among 4,204 SM patients diagnosed between 2004 and 2016, of which $0.8 \%$ were removed $(3,396)[18]$. With the SEER, Westwick and Shamji [3] found an age-adjusted incidence of 0.193 per 100,000 population ( $95 \%$ CI, $0.183-0.202$ ).

Using a different database (Central Brain Tumor Registry of the United States [CBTRUS]), Kshettry et al. [19] found 7,148 newly diagnosed SM between 2004 and 2010, resulting in an overall age-adjusted incidence of 0.33 per 100,000 population, which was relatively stable over the study period. They noticed the highest incidence in the

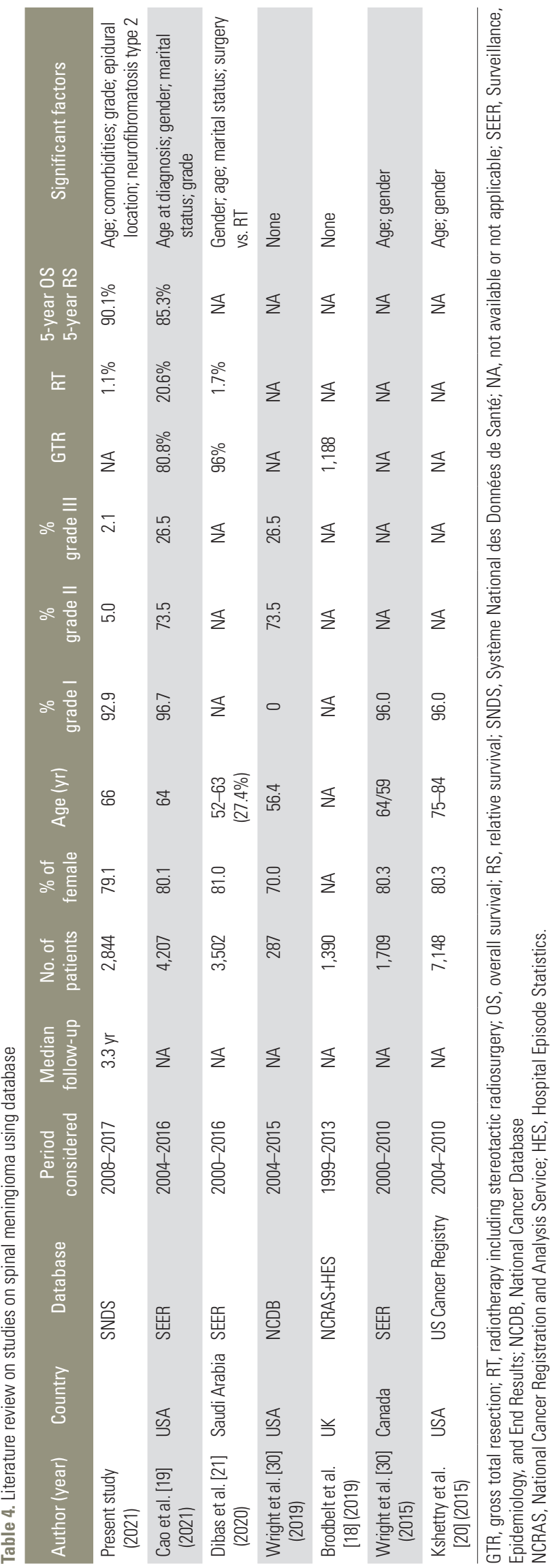


75- to 84-year-old age group and a slightly lower gender ratio of 3.37 [19]. However, only $85.5 \%$ of the SM were removed $(n=6,112)$ and microscopically confirmed with $96.1 \%$ of benign WHO I meningioma, $2.5 \%$ of grade II, and $1.4 \%$ of malignant meningioma. These rates correspond somewhat to the findings of $92.9 \%, 5 \%$, and $2.1 \%$, respectively, of the current study. However, a significant difference still exists $(p<0.001)$. In addition, psammomatous meningioma is the most frequent histopathological subtype [18].

The strengths of the SNDS reside both in the high number of patients and in the exhaustive data available from both private and public hospitals in France. The SNDS includes much information (e.g., demographic data, medical and surgical procedures with linked and associated diagnoses, and date of expiry). The database representativeness is nearly perfect because it includes the whole country's population of nearly 68 million inhabitants constituting one of the largest AMDB in the world [11].

Only a handful of studies reporting on SM use AMDB, which all exploit American databases (e.g., SEER and/or CBTRUS) [3,18-20]. Compiled from a number of institutions, SNDS accuracy is nonetheless limited by inconstancies in data collection and recording. Despite some limitations, the SNDS is an invaluable tool to assess meningioma outcomes. It offers an incomparable means to explore associations with other pathology, medication, or combined surgical treatment that has and could not be previously assessed. The retrospective nature of this study, together with the lack of clarity regarding treatment rationales and nonhomogeneous management strategies without random assignment, needs to be considered when evaluating the results. Moreover, important variables such as the quality of resection are not recorded in the SNDS [21].

The primary goal of surgery is to achieve complete tumor removal with or without dural excision while avoiding additional neurological damage. Radiotherapy or stereotactic radiosurgery may be given for residual or recurrent disease or higher-grade tumors. SM resection is usually a relatively simple neurosurgical intervention with reported rates of complete resection (Simpson grade I or II) often $>90 \%[22,23]$. Such a procedure is associated with low morbidity and rare mortality. Compared to intracranial meningioma, aggressive SMs are infrequent. Moreover, recurrence is uncommon once removed. Brodbelt et al. [17] noted that OS after surgery is better in women, younger adults, and people with SM and that patients with SM did better in all grades, gender, and ages.

Few studies analyzed the survival of SM patients [18]. Cao et al. [18]'s reported 5- and 10-year rates of $85.3 \%$ and $70.9 \%$, respectively, are surprisingly quite low for a generally benign tumor not supposed to threaten the life of the affected patients. Throughout the studies, patients with SM were even more likely to be older than those with intracranial meningioma with reported age at surgery often $>65$ years and thus reaching life expectancy. Derived from an unselected sample, the result of the current study indicates that SM is not a cause of mortality with a 5-year survival relative to the expected survival of the age- and gender-matched French standard population of $100.6 \%$ (95\% CI, 98.6\%-102.5\%). Brodbelt et al. [17] also found a 5 -year net survival of $98 \%$, asserting that SM surgery insignificantly contributes to the overall mortality. In standard survival analysis, subjects are supposed to experience only one type of event, commonly recurrence or expiry. In reality, several types may occur. In these cases, other events-so-called competing events-may preclude the occurrence of the event of interest or modify the risk that occurs in the primary endpoint. The traditional methods of survival analysis (e.g., the Kaplan-Meier method and the Cox proportional hazards model) are not designed to accommodate the competing nature of multiple events assuming the absence of competing risks. Net survival describes the probability of surviving a tumor diagnosis in the absence of competing causes of mortality and is defined as the survival that may occur if all risks of expiry from other causes rather than the disease of interest, meningioma in this study, were removed. Net survival is now a major epidemiological indicator, already routinely estimated for many neoplasms by either cause-specific or RS. The first one requires knowing the cause of expiry. However, when causes of expiry are unavailable or unreliable, net survival may be assessed by a measure of the RS, which uses the all-cause mortality of the study group and the expected mortality of a disease-free group having the same demographic characteristics. RS is thus calculated as the observed OS in the meningioma cohort relative to that expected in the general population [24]. Derived from an unselected sample, this study indicates that SM is a not a cause of mortality in the affected patient population. If indicated, SM surgery should thus be performed regardless of the age of the patient. The results of the survival analysis also confirmed that the patients had better outcomes if surgery was performed [18]. 
Several factors are associated with SM survival (e.g., age, gender, size, and gross total resection) [3,18,19,21,23,25] (Table 4). For Cao et al. [18], younger patients with benign meningiomas have a longer survival. Likewise, in the current study, malignant meningioma were associated to a reduced survival (HR, 2.64; 95\% CI, 1.51-4.61; $p<0.001)$ (Fig. 1D). However, females were not found to have a better prognosis compared with males $[3,18]$. Unsurprisingly, the level of comorbidities did influence OS (HR, 1.44; 95\% CI, 1.34-1.54; $p<0.001)$. Meningiomas are the second most frequent tumor type in NF2 with a prevalence between $53 \%$ and $66 \%$ [26]. The cumulative incidence of meningiomas was shown to be close to $80 \%$ by 70 years old in a cohort of 411 patients with proven NF2 mutation [27]. In a previous report, the median age of expiry of NF2 patients was found to be 41.7 years (IQR, 32.7-50.4 years), and $15.2 \%$ had SM surgery [4]. The current study confirms the shorter survival of such patients $(\mathrm{HR}, 3.65$; 95\% CI, 1.28-10.39; $p=0.0152$ ).

One of the advantages of the SNDS, which uses the CCAM classification, is to provide the precise location of meningioma dural insertion, which is an invaluable feature. Most SMs are removed by a posterior or posterolateral approach without the need for fixation, which was only necessary for $0.7 \%$ in the current series [28]. A hemilaminectomy approach, which was quick and safe with minimal morbidity and spinal instability, was usually performed to remove SM [29]. Approximately, an extradural component is seen but an exclusively extradural SM is quite uncommon in $10 \%$ of cases [30]. Epidural SM was found solely in $6.9 \%$ within the current cohort. However, they are associated with a worse prognosis (HR, 1.73; 95\% CI, $1.09-2.75 ; p=0.0206)$. Medical literature on epidural $\mathrm{SM}$ is scarce, and any satisfactory reason to explain this impaired OS was not found.

This work highlights the great value of this unique database to evaluate outcomes after SM surgery. Further inclusion and prolonged follow-up are required to assess other predictors (e.g., gender, EBRT, or histopathological subtypes) best after SNDS and the French Brain Tumour Database merging [6].

\section{Conclusions}

The SNDS is of great value to assess SM incidence, associated mortality, and its predictors. OS after meningioma surgery is favorable but may be impaired for NF2 or older patients with a high level of comorbidities, epidural tumor, and malignant histopathology. Performing SM surgery on even more senior patients compared with intracranial meningioma is not associated with an increased absolute excess risk of expiry.

\section{Conflict of Interest}

No potential conflict of interest relevant to this article was reported.

\section{Acknowledgments}

Marjorie Boussac and Julius Kemme from the CNAM for their help in data extraction.

\section{References}

1. Ostrom QT, Patil N, Cioffi G, Waite K, Kruchko C, Barnholtz-Sloan JS. CBTRUS statistical report: primary brain and other central nervous system tumors diagnosed in the United States in 2013-2017. Neuro Oncol 2020;22(12 Suppl 2):iv1-96.

2. Champeaux C, Weller J, Katsahian S. Epidemiology of meningiomas: a nationwide study of surgically treated tumours on French medico-administrative data. Cancer Epidemiol 2019;58:63-70.

3. Westwick HJ, Shamji MF. Effects of sex on the incidence and prognosis of spinal meningiomas: a surveillance, epidemiology, and end results study. J Neurosurg Spine 2015;23:368-73.

4. Champeaux-Depond C, Weller J, Resche-Rigon M. Neurofibromatosis type 2: a nationwide populationbased study focused on survival after meningioma surgery. Clin Neurol Neurosurg 2020;198:106236.

5. Champeaux C, Houston D, Dunn L, Resche-Rigon M. Intracranial WHO grade I meningioma: a competing risk analysis of progression and disease-specific survival. Acta Neurochir (Wien) 2019;161:2541-9.

6. Zouaoui S, Darlix A, Rigau V, et al. Descriptive epidemiology of 13,038 newly diagnosed and histologically confirmed meningiomas in France: 2006-2010. Neurochirurgie 2018;64:15-21.

7. Champeaux C, Jecko V, Houston D, et al. Malignant meningioma: an international multicentre retrospective study. Neurosurgery 2019;85:E461-9.

8. Champeaux C, Wilson E, Brandner S, Shieff C, 
Thorne L. World Health Organization grade III meningiomas: a retrospective study for outcome and prognostic factors assessment. Br J Neurosurg 2015;29:693-8.

9. Champeaux C, Houston D, Dunn L. Atypical meningioma: a study on recurrence and disease-specific survival. Neurochirurgie 2017;63:273-81.

10. Champeaux C, Dunn L. World Health Organization grade II meningioma: a 10-year retrospective study for recurrence and prognostic factor assessment. World Neurosurg 2016;89:180-6.

11. Tuppin P, Rudant J, Constantinou P, et al. Value of a national administrative database to guide public decisions: from the système national d'information interrégimes de l'Assurance Maladie (SNIIRAM) to the système national des données de santé (SNDS) in France. Rev Epidemiol Sante Publique 2017;65 Suppl 4:S149-67.

12. Champeaux-Depond C, Weller J, Froelich S, RescheRigon M. A nationwide population-based study on overall survival after meningioma surgery. Cancer Epidemiol 2021;70:101875.

13. Constantinou P, Tuppin P, Fagot-Campagna A, Gastaldi-Menager C, Schellevis FG, Pelletier-Fleury $\mathrm{N}$. Two morbidity indices developed in a nationwide population permitted performant outcome-specific severity adjustment. J Clin Epidemiol 2018;103:6070.

14. R Core Team. R: a language and environment for statistical computing. Vienna: R Foundation for Statistical Computing; 2014.

15. Lang TA, Altman DG. Basic statistical reporting for articles published in biomedical journals: the "Statistical Analyses and Methods in the Published Literature" or the SAMPL Guidelines. Int J Nurs Stud 2015;52:5-9.

16. Nicholls SG, Quach P, von Elm E, et al. The Reporting of Studies Conducted Using Observational Routinely-Collected Health Data (RECORD) statement: methods for arriving at consensus and developing reporting guidelines. PLoS One 2015;10:e0125620.

17. Brodbelt AR, Barclay ME, Greenberg D, Williams M, Jenkinson MD, Karabatsou K. The outcome of patients with surgically treated meningioma in England: 1999-2013: a cancer registry data analysis. Br J Neurosurg 2019;33:641-7.
18. Cao Y, Jiang Y, Liu C, et al. Epidemiology and survival of patients with spinal meningiomas: a SEER analysis. Eur J Surg Oncol 2021;47:2340-5.

19. Kshettry VR, Hsieh JK, Ostrom QT, Kruchko C, Benzel EC, Barnholtz-Sloan JS. Descriptive epidemiology of spinal meningiomas in the United States. Spine (Phila Pa 1976) 2015;40:E886-9.

20. Dibas M, Rajab AM, Atiah MJ, et al. Racial disparities in the incidence and survival of spinal meningioma. Asian J Neurosurg 2020;15:877-81.

21. Simpson D. The recurrence of intracranial meningiomas after surgical treatment. J Neurol Neurosurg Psychiatry 1957;20:22-39.

22. Hua L, Zhu H, Deng J, et al. Clinical and prognostic features of spinal meningioma: a thorough analysis from a single neurosurgical center. J Neurooncol 2018;140:639-47.

23. Jamilson Araujo Pereira B, Nogueira de Almeida A, Silva Paiva W, Henrique Pires de Aguiar P, Jacobsen Teixeira M, Kazue Nagahashi Marie S. Neuro-oncological features of spinal meningiomas: systematic review. Neurochirurgie 2020;66:41-4.

24. Pohar M, Stare J. Relative survival analysis in R. Comput Methods Programs Biomed 2006;81:272-8.

25. Wright JM, Wright CH, Cioffi G, et al. Survival in patients with high-grade spinal meningioma: an analysis of the National Cancer Database. World Neurosurg 2019;129:e749-53.

26. Asthagiri AR, Parry DM, Butman JA, et al. Neurofibromatosis type 2. Lancet 2009;373:1974-86.

27. Goutagny S, Kalamarides M. Meningiomas and neurofibromatosis. J Neurooncol 2010;99:341-7.

28. Misra SN, Morgan HW. Avoidance of structural pitfalls in spinal meningioma resection. Neurosurg Focus 2003;14:e1.

29. Turel MK, D'Souza WP, Rajshekhar V. Hemilaminectomy approach for intradural extramedullary spinal tumors: an analysis of 164 patients. Neurosurg Focus 2015;39:E9.

30. Pandey S, Singh K, Sharma V, Ghosh A, Suman S. Dorsal spinal epidural psammomatous meningioma in an adult male. J Neurosci Rural Pract 2016;7:125-7. 